\title{
Stability and Gait Planning of 3-UPU Hexapod Walking Robot
}

\author{
Ruiqin $\mathrm{Li}^{1, *}$, Hongwei Meng ${ }^{1}$, Shaoping Bai ${ }^{2}{ }^{(\mathbb{D}}$, Yinyin Yao ${ }^{1}$ and Jianwei Zhang ${ }^{1,3}$ \\ 1 School of Mechanical Engineering, North University of China, Taiyuan 030051, China; \\ menghw@st.nuc.edu.cn (H.M.); Yao_yinyin@st.nuc.edu.cn (Y.Y.); zhang@informatik.uni-hamburg.de (J.Z.) \\ 2 Department of Mechanical and Manufacturing Engineering, Aalborg University, 9220 Aalborg, Denmark; \\ shb@mp.aau.dk \\ 3 Department of Informatics, University of Hamburg, 22527 Hamburg, Germany \\ * Correspondence: liruiqin@nuc.edu.cn; Tel.: +86-351-3921300
}

Received: 26 July 2018; Accepted: 29 August 2018; Published: 31 August 2018

\begin{abstract}
The paper presents an innovative hexapod walking robot built with 3-UPU parallel mechanism. In the robot, the parallel mechanism is used as both an actuator to generate walking and also a connecting body to connect two groups of three legs, thus enabling the robot to walk with simple gait by very few motors. In this paper, forward and inverse kinematics solutions are obtained. The workspace of the parallel mechanism is analyzed using limit boundary search method. The walking stability of the robot is analyzed, which yields the robot's maximum step length. The gait planning of the hexapod walking robot is studied for walking on both flat and uneven terrains. The new robot, combining the advantages of parallel robot and walking robot, has a large carrying capacity, strong passing ability, flexible turning ability, and simple gait control for its deployment for uneven terrains.
\end{abstract}

Keywords: hexapod walking robot; 3-UPU parallel mechanism; kinematics; stability; gait planning

\section{Introduction}

Legged robots have advantages over the wheeled robots in their flexibility of movement and the adaptability of the environment. Legged robots can adapt to different terrains. A multi-DOF design enables it to actively adjust the height of the body according to the operational requirements to ensure the balance and stability of the body.

To date, many legged robots have been developed [1-4]. The NOROS robot developed by Ding et al. [2,3] is a modular walking robot. It consists of up to eight autonomous leg modules, each equipped with various sensors. A self-recovery approach is applied by imitating the self-recovery motion of insects. Ma et al. [4] proposed a robot which consists of six legs of the same structure distributed evenly on a platform. Each leg of the robot has a structure similar to that of a telescopic parallelogram mechanism with folding capability, which is beneficial to the gait planning and real-time control of the robot. The parallel type mobile robot formed by parallel mechanism applied to the legged robot can greatly improve the load/weight ratio of the mobile robot, reduce the energy consumption, and prolong the walking time. Our interest is to develop a hexapod walking robot that utilizes a parallel mechanism, namely, a three-DOF translational 3-UPU parallel mechanism.

The concept of 3-UPU parallel mechanism was first introduced by Tsai [5], which can produce three-dimensional translation movement. Gregorio [6] presented a 3-UPU parallel manipulator, named 3-UPU wrist for spherical motions with its prismatic pairs actuated. Ji et al. [7] proposed a 3-UPU translational parallel robotic manipulator with an equal offset in its six universal joints and obtained 16 solutions for forward kinematics. Han et al. [8] analyzed kinematic sensitivity of the 3-UPU parallel 
mechanism. Huang et al. [9] studied feasible instantaneous motions and kinematic characteristics of an orthogonal 3-UPU parallel mechanism. Wu et al. [10] designed a configuration-switch mechanism for 3-UPU parallel mechanism.

The kinematics and dynamics analyses of 3-UPU parallel mechanisms have been well developed in literatures [11-15]. Two novel 3-UPU parallel kinematics machines with two rotations and one translation are proposed in literature [13]. In addition, singularity [14,15] and stiffness [16] of the 3-UPU parallel mechanism are also studied.

There are some reports about applications of parallel mechanism in mobile robots. A rolling biped robot was proposed based on a 3-UPU parallel mechanism [17]. Gait and stability analyses were presented and four rolling modes of the mechanism were discussed and simulated. Gu [18] proposed to apply a typical 3-UPU parallel mechanism in a quadrupedal walking robot. Wang et al. [19] presented a bipedal locomotor consisting of two identical 3-DOF tripod leg mechanisms with a parallel manipulator architecture. Sugahara et al. [20] presented a design of a battery driven bipedal robot, which used 6-DOF parallel mechanism for its each leg. Wang et al. [21] proposed a quadruped/biped reconfigurable parallel legged walking robot, in which the robot can change between biped and quadruped walking modes according to real-time road conditions. Each leg is composed of a 3-UPU parallel mechanism. The robot walks as a quadruped generally and changes to bipedal walking when walking up and down stairs.

Walking robots have to walk following a certain pattern of leg movement, namely, the gaits. Hirakoso et al. [22] developed a multi-legged gait prototype robot with four legs consisted of redundant joint. An optimal control system was proposed to control any motion for the four-legged robot with redundant joint. Sun et al. [23] proposed a transformable wheel-legged mobile robot that integrated stability and maneuverability of wheeled robot and obstacle climbing capability of legged robot. When under wheeled mode, the robot avoids the obstacle using a motion control strategy that combines three basic cases of translation, rotation, and arc motion, while the robot climbs the obstacle by legs. A method of free gait generation that utilizes the primary/secondary gait for both straight line and circular body trajectories was proposed by Bai [24]. Four constraints of primary gait were discussed. When the walking machine cannot move using the primary gait, the secondary gait is generated to adjust the leg position and enable the vehicle to keep on moving. Gait planning combined with path planning was also developed $[25,26]$.

Gong et al. [27] focused on the dynamic gaits control for complex robot with 20 DOFs. Using composite cycloid to plan the swing foot trajectory curve made the velocity and acceleration to be zero which can reduce the impact of collision with ground and energy loss. Li et al. [28] presented a three-dimensional model of a quadruped robot which has six DOFs on torso and five DOFs on each leg. Matsuzawa et al. [29] proposed a crawling motion to reduce the risk of malfunction due to falling when a legged robot travels across rough terrain.

Winkler et al. [30,31] developed trajectory planning for legged locomotion that automatically determines the gait sequence, step timings, footholds, swing-leg motions, and six-dimensional body motion over rough terrain, without any additional modules. Neunert et al. [32] proposed a trajectory optimization framework for whole-body motion planning through contacts. Zhao et al. [33] developed a motion generation approach for a hexapod robot Octopus-III to control the robot to walk along the planned trajectory. The approach coordinates the body motion and the feet motions to fulfill requirements of walking stability and kinematic feasibility simultaneously. Oliveira et al. [34] studied locomotion patterns of hexapod robots, including metachronal wave gait, and tetrapod and tripod gaits.

Up to date, the reported research works of legged robots are mainly focused on biped, quadruped, and hexapod robots. Most of these robots have independent driving joints on the legs, which requires many actuators. In the case of a single leg of three joints, a hexapod robot needs at least 18 motors which greatly increases the weight of the robot. Moreover, the position of each foot has to be considered, which adds complexity to control. 
The paper presents a hexapod walking robot designed with a 3-UPU parallel mechanism, aiming to reduce the number of driving motors and improve load capacity and terrain adaptability. The new design is characterized by alternating motion between the two platforms of the 3-UPU parallel mechanism to realize robot walking. The parallel mechanism is used as both an actuator to generate walking and also a connecting body to connect two groups of three legs. The new walking robot is thus able to walk with simple gait by very few motors.

The paper is organized as follows. Firstly, the configuration of the hexapod walking robot is presented in Section 2. The forward and inverse kinematic solutions of the 3-UPU parallel mechanism are derived in Section 3. The workspace of the robot is obtained using joint constraint conditions and inverse kinematics equation in Section 4. The movement stability of the robot is analyzed using the center of gravity projection method in Section 5 . The gait of the robot is planned under the conditions of ensuring the efficiency of the walking mode of the robot in Section 6. A case study is included in Section 7 , and the work is concluded in Section 8.

\section{Configuration of 3-UPU Hexapod Walking Robot}

A 3-UPU hexapod walking robot is proposed, as shown in Figure 1a. The 3-UPP mechanism, shown separately in Figure 1b, connects two platforms. On each platform, three retractable legs, distributed evenly on the triangular platforms, are mounted to support the robot. Servo motors are mounted at the end of each supporting leg to adjust its length in fitting uneven ground. A separate pressure sensor is installed on each supporting leg to detect whether the supporting leg is in contact with the ground.

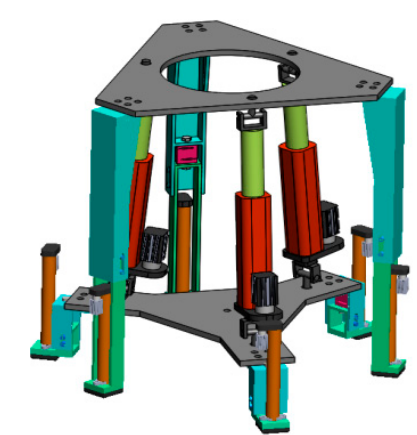

(a)

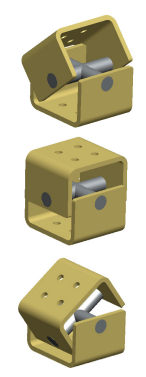

Swinging in one direction

Swinging direction switching

Swinging in another direction

(c)

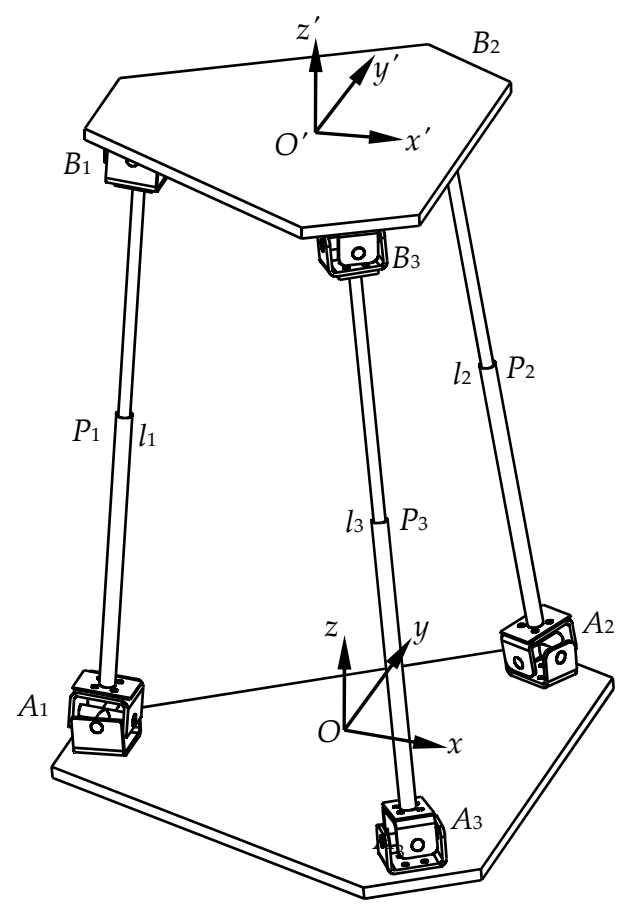

(b)

Figure 1. Structure of 3-UPU hexapod walking robot. (a) 3D model. (b) 3-UPU parallel mechanism. (c) The orientation of the Hooke joint.

The upper and lower platforms of the 3-UPU parallel mechanism are connected by three limbs. The structures of the Hooke joints in three limbs are different. In two of these limbs, the structures of the Hooke joints are the same, which can supply two rotational DOFs. In another limb, two forks of the Hooke joint are just in contact with each other, as shown in Figure 1c. The active fork can only 
swing in one direction during the movement of the robot. The swinging direction can be switched when the active fork swings to its highest position. This structure of the Hooke joint can effectively control the moving direction of 3-UPU hexapod walking robot.

The rotating shafts on forks fixed to the platform are tangent to the circumcircle of the equilateral triangle formed by mounting positions of three Hooke joints. Because there are three couples which limit the three rotational DOFs of the mechanism, thus the moving platform has only three translational DOFs.

\section{Kinematics of 3-UPU Parallel Mechanism}

We briefly describe the kinematics of 3-UPU parallel mechanism, upon which the locomotion kinematics is developed.

\subsection{Inverse Kinematics Solution}

The moving, or the upper platform of 3-UPU parallel mechanism is a mechanism with only three-dimensional translations. The inverse kinematics problem of 3-UPU parallel mechanism is to solve the lengths $\left(l_{1}, l_{2}, l_{3}\right)$ of the equivalent driving links for given position $(x, y, z)$ of the moving platform.

As shown in Figure $1 \mathrm{~b}$, the moving coordinate system $O^{\prime}-x^{\prime} y^{\prime} z^{\prime}$ is connected to the moving platform. The origin $O^{\prime}$ is located at the center of the moving platform. $x^{\prime}$ axis is coincident with $O^{\prime} B_{1}$, and point $B_{1}$ is located at the negative direction of $x^{\prime}$ axis. $y^{\prime}$ axis is parallel to line $B_{3} B_{2} . z^{\prime}$ axis is perpendicular to the upper platform and pointing away from the mechanism. The coordinate system $O-x y z$ is connected to the lower, or the static platform. The origin $O$ is located at the center of the static platform. The $x$ axis is coincident with $O A_{1}$, and point $A_{1}$ is located at the axis' negative part. $y$ axis is parallel to line $A_{3} A_{2} . z$ axis is perpendicular to the static platform and the direction is upward. Moreover, the circumradii of the moving platform and the static platform are noted as $r$ and $R$, respectively. The equivalent link lengths of the limb $i$ are noted by $l_{i}(i=1,2,3)$.

According to the geometric characteristics of the mechanism, the closed vector loop equation is established as:

$$
\begin{gathered}
A_{i} B_{i}=-O A_{i}+O O^{\prime}+O^{\prime} B_{i},(i=1,2,3) \\
\left|A_{i} B_{i}\right|=l_{i},(i=1,2,3)
\end{gathered}
$$

Let the coordinate of the geometric center $O^{\prime}$ in the static coordinate system $O-x y z$ be $(x, y, z)$. The vector $\boldsymbol{O O}^{\prime}$ can be expressed as:

$$
\boldsymbol{O O}^{\prime}=\left[\begin{array}{lll}
x, & y, & z
\end{array}\right]^{\mathrm{T}}
$$

and $\boldsymbol{O} \boldsymbol{A}_{\boldsymbol{i}}$ and $\boldsymbol{O}^{\prime} \boldsymbol{B}_{\boldsymbol{i}}(i=1,2,3)$ can be expressed as:

$$
\begin{array}{cc}
\boldsymbol{O} \boldsymbol{A}_{\mathbf{1}}=\left[\begin{array}{lll}
-R, & 0, & 0
\end{array}\right]^{\mathrm{T}} & \boldsymbol{O}^{\prime} \boldsymbol{B}_{\mathbf{1}}=\left[\begin{array}{lll}
-r, & 0, & 0
\end{array}\right]^{\mathrm{T}} \\
\boldsymbol{O} \boldsymbol{A}_{\mathbf{2}}=\left[\begin{array}{lll}
\frac{1}{2} R, & \frac{\sqrt{3}}{2} R, & 0
\end{array}\right]^{\mathrm{T}} & \boldsymbol{O}^{\prime} \boldsymbol{B}_{2}=\left[\begin{array}{lll}
\frac{1}{2} r, & \frac{\sqrt{3}}{2} r, & 0
\end{array}\right]^{\mathrm{T}} \\
\boldsymbol{O} \boldsymbol{A}_{\mathbf{3}}=\left[\begin{array}{lll}
\frac{1}{2} R, & -\frac{\sqrt{3}}{2} R, & 0
\end{array}\right]^{\mathrm{T}} & \boldsymbol{O}^{\prime} \boldsymbol{B}_{3}=\left[\begin{array}{lll}
\frac{1}{2} r, & -\frac{\sqrt{3}}{2} r, & 0
\end{array}\right]^{\mathrm{T}}
\end{array}
$$

From Equations (1), (3), and (4), the following expressions can be obtained.

$$
\begin{gathered}
A_{1} B_{1}=\left[\begin{array}{lll}
x+\Delta, & y, & z
\end{array}\right]^{\mathrm{T}} \\
A_{2} B_{2}=\left[\begin{array}{lll}
x-\frac{1}{2} \Delta, & y-\frac{\sqrt{3}}{2} \Delta, & z
\end{array}\right]^{\mathrm{T}} \\
A_{3} B_{3}=\left[\begin{array}{lll}
x-\frac{1}{2} \Delta, & y+\frac{\sqrt{3}}{2} \Delta, & z
\end{array}\right]^{\mathrm{T}}
\end{gathered}
$$

where $\Delta=R-r$. 
According to Equation (2), the position inverse solution can be obtained.

$$
\left\{\begin{array}{l}
(x+\Delta)^{2}+y^{2}+z^{2}=l_{1}{ }^{2} \\
\left(x-\frac{1}{2} \Delta\right)^{2}+\left(y-\frac{\sqrt{3}}{2} \Delta\right)^{2}+z^{2}=l_{2}{ }^{2} \\
\left(x-\frac{1}{2} \Delta\right)^{2}+\left(y+\frac{\sqrt{3}}{2} \Delta\right)^{2}+z^{2}=l_{3}{ }^{2}
\end{array}\right.
$$

When the basic geometric dimensions of the 3-UPU parallel mechanism and the position $(x, y, z)$ of the moving platform are known, the variation of the equivalent link length $l_{i}(i=1,2,3)$ of three limbs, i.e., the displacement of three prismatic pairs can be obtained using Equation (6). Similarly, when the upper platform is a static platform and the lower platform is a moving platform, the static coordinate system $O-x y z$ is connected to the upper platform, while the moving coordinate system $O^{\prime}-x^{\prime} y^{\prime} z^{\prime}$ is connected to the lower platform. The coordinate directions do not change, and the closed vector loop equation is established as:

$$
B_{i} A_{i}=-O B_{i}+O O^{\prime}+\boldsymbol{O}^{\prime} A_{i}(i=1,2,3)
$$

Similarly, the inverse position solution of the upper platform can be obtained as:

$$
\left\{\begin{array}{l}
(x-\Delta)^{2}+y^{2}+z^{2}=l_{1}{ }^{2} \\
\left(x+\frac{1}{2} \Delta\right)^{2}+\left(y+\frac{\sqrt{3}}{2} \Delta\right)^{2}+z^{2}=l_{2}{ }^{2} \\
\left(x+\frac{1}{2} \Delta\right)^{2}+\left(y-\frac{\sqrt{3}}{2} \Delta\right)^{2}+z^{2}=l_{3}{ }^{2}
\end{array}\right.
$$

\subsection{Kinematics Forward Solution}

Giving the lengths $\left(l_{1}, l_{2}, l_{3}\right)$ of the prismatic pairs of three limbs, we need to solve the spatial position $(x, y, z)$ of the moving platform. The solutions of position $(x, y, z)$ can be obtained numerically using Equation (6).

\section{Workspace of the 3-UPU Parallel Mechanism}

\subsection{Factors Influencing the Workspace of the 3-UPU Parallel Mechanism}

The reachable workspace of the 3-UPU parallel mechanism is under the influence of several factors: the shortest and longest distance of the limb and the range of rotation angle of Hooke joint.

\subsubsection{Shortest and Longest Distances of the Limbs}

The position $(x, y, z)$ of the reference point of the moving platform is constrained by the equivalent link length $l_{i}(i=1,2,3)$ of the three limbs. The three prismatic pairs in the limbs act as driving inputs, which makes the moving platform move in the workspace range. When the limb is the shortest or the longest, servo motor I, which acts as the prismatic pair, is in the zero position or the limit position. The reference point of the moving platform reaches the boundary of the workspace.

Taking the upper platform as the moving platform, the equivalent link length $l_{i}(i=1,2,3)$ of each limb satisfy the constraint:

$$
l_{\min }<l_{i}<l_{\max }
$$

where $l_{\min }$ and $l_{\max }$ are the minimum and maximum link lengths of the limb. 


\subsubsection{Rotation Angle Range of the Hooke Joint}

The Hooke joints are used to connect the limbs to the upper and lower platforms. As shown in Figure 2, the rotation angle $\theta_{1}$ of the Hooke joint connected to the limb is related to the length $l_{1}$ of the limb and the $y$ coordinate of the moving platform. The rotation angle $\theta_{1}$ should satisfy:

$$
\theta_{1}=\arcsin \frac{B_{1} E}{l_{1}}=\arcsin \frac{|y|}{l_{1}}
$$

The rotation angle $\theta_{2}$ of the Hooke joint connected to the static platform is related to the projection length of the limb $l_{1}$ at $O-x z$ plane and the $z$ coordinate of the moving platform. The rotation angle $\theta_{2}$ should satisfy:

$$
\theta_{2}=\arccos \frac{E F}{A_{1} E}=\arccos \frac{z}{\sqrt{l_{1}^{2}-y^{2}}}
$$

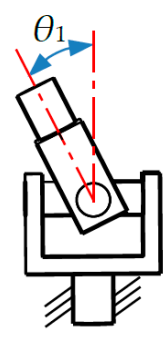

(a)

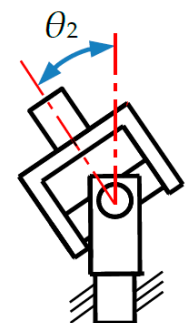

(b)

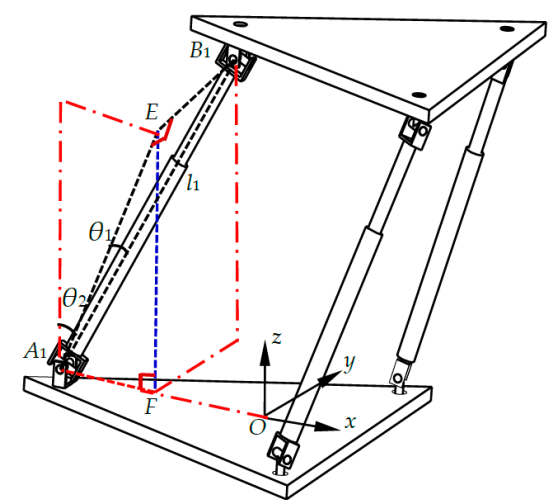

(c)

Figure 2. Rotation angles of the Hooke joint in the 3-UPU parallel mechanism. (a) Angle $\theta_{1}$ of the Hooke joint. (b) Angle $\theta_{2}$ of the Hooke joint. (c) Rotation angles of the Hooke joint.

\subsection{Method for Determining Workspace of the 3-UPU Parallel Mechanism}

The workspace of the 3-UPU parallel mechanism is determined using the limit boundary search method. The idea is that when the position of the moving platform is known, the equivalent link length $l_{i}(i=1,2,3)$ of each limb can be calculated by the inverse solution equation of the mechanism position. The rotation angles of Hooke joint can be solved using Equations (10) and (11). Then, these results are compared with the corresponding limit values, respectively. If any of these values exceed the allowable range, that is, the reference point is outside the workspace. The solutions are

(1) Defining the structure parameter of the mechanism, including: the circumradii $r$ and $R$ of the upper and lower platforms, the maximum travel range of the equivalent link length $l_{i}(i=1,2,3)$ of limb $i$, the maximum rotation angle of Hooke joint.

(2) Defining the range of the coordinates $(x, y, z)$ of the reference points of the moving platform, or, the search space. 
(3) The reference point coordinate is substituted into the position inverse solution equation of the mechanism, the equivalent link length $l_{i}(i=1,2,3)$ and the Hooke joint angles $\theta_{1}$ and $\theta_{2}$ in the limbs are obtained. The results are checked whether they are within the allowable range. If they are, these points are recorded.

(4) The set of points satisfying the condition is the workspace of the 3-UPU parallel mechanism when a given range of values has been searched.

The design parameters are given in Table 1. According to workspace search flow chart in Figure 3, the workspaces of the 3-UPU parallel mechanism are obtained using MATLAB.

Table 1. Design parameters of the 3-UPU parallel mechanism.

\begin{tabular}{cc}
\hline Parameter & Symbol and Unit \\
\hline Equivalent circumradius of the upper platform & $r / \mathrm{mm}$ \\
Equivalent circumradius of the lower platform & $R / \mathrm{mm}$ \\
Range of the equivalent link length of limb $i$ & $l_{i}(i=1,2,3) / \mathrm{mm}$ \\
Range of the rotation angle of the Hooke joint & $\theta_{i}(i=1,2) / \mathrm{rad}$ \\
\hline
\end{tabular}

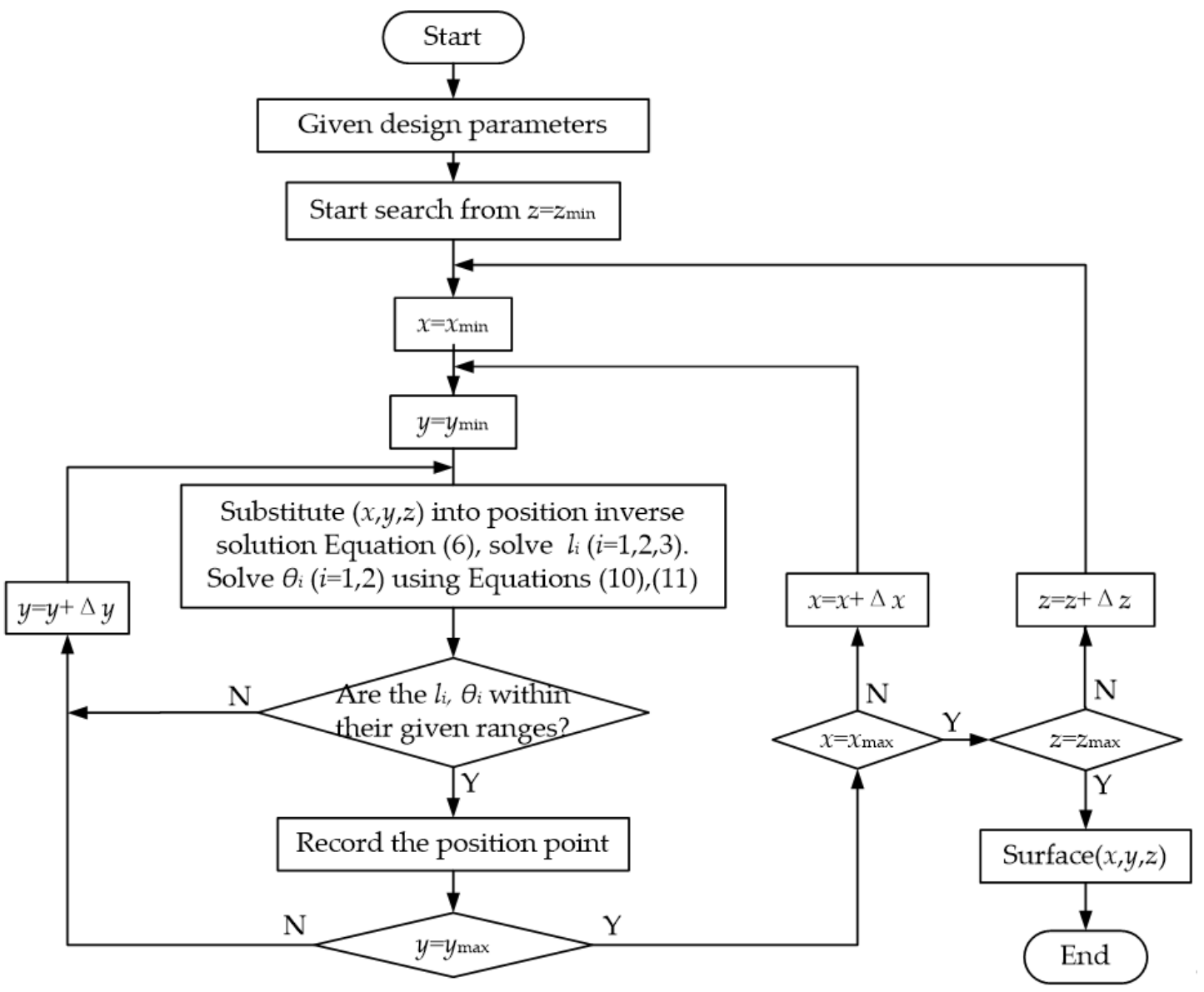

Figure 3. Workspace search flow chart for the 3-UPU parallel mechanism.

\section{Stability of 3-UPU Hexapod Walking Robot}

The walking of 3-UPU hexapod robot is realized by alternative shifting between the upper and lower platforms, each with three retractable legs to support the whole robot. During the movement of the robot, the robot is supported by three legs and six legs alternately. An overturn can happen in the phase of three-leg support. Stability analysis is thus needed. 
Stability of walking robots can be analyzed in terms of static and dynamic stability. A walking robot is statically stable if the horizontal projection of its center of gravity lies inside the support polygon, while it is dynamically stable when the robot walking is stable even if the static stable condition is not satisfied. As the 3-UPU hexapod robot walks in a relative slow speed, we analyze only its static stability.

As shown in Figure 4, three points-E, $F, H$-are the landing positions of the three supporting legs of lower platform, respectively. They form a supporting triangle. Let the center of gravity of the upper platform and its three legs be marked as $G_{1}$, the center of gravity of the lower platform and its three legs as $G_{2}$. The center of gravity of the whole robot, located on the line connecting two points $G_{1}$ and $G_{2}$, is marked as $G_{3}$. Figure 5 shows the relation between the projection of the center of gravity and stability margin $d$. When the projection of the robot's center of gravity $G_{3}$ on the ground is located within the supporting triangle, the walking is stable. Otherwise, the walking of the robot is unstable and the robot is likely overturned.

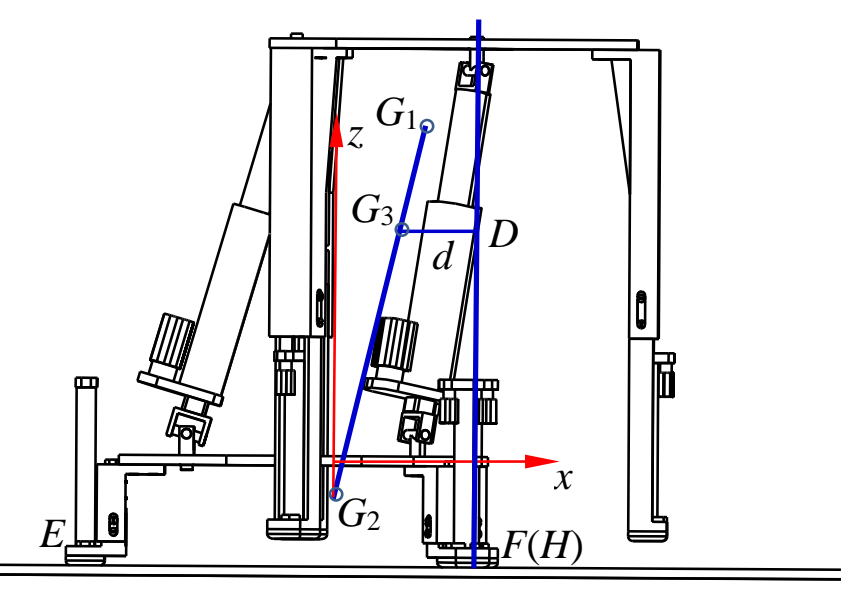

Figure 4. Three centers of gravity considered in the stability analysis.

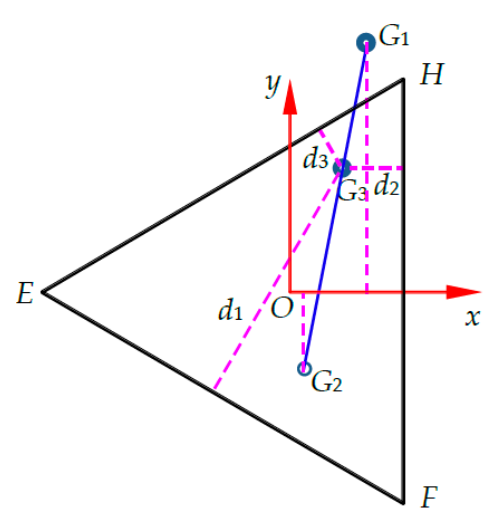

(a)

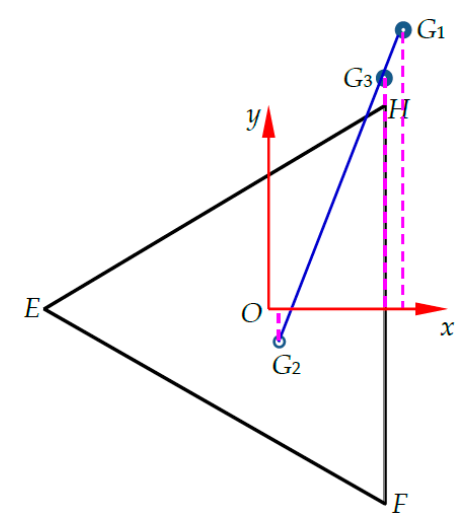

(b)

Figure 5. Relation between the projection of the center of gravity and stability margin. (a) The projection of the center of gravity is located within the supporting triangle. (b) The projection of the center of gravity is located on the boundary of the supporting triangle.

The projections of points $G_{1}$ and $G_{2}$ are coincident in the initial status, that is the six legs of the robot land on the ground. When the projection of the center of gravity $G_{3}$ moves inside the supporting triangle, the amount of movement allowed is the smallest in the direction perpendicular to the boundary. Thus, the displacement of the projection of the center of gravity $G_{3}$ in the $O-x y$ plane is analyzed. As shown in Figure 5 a, let the horizontal coordinate of points $G_{1}$ and $G_{3}$ be $x_{G 1}$ and $x_{G 3}$, 
respectively, the mass of the upper platform and its three legs be $m_{1}$, the mass of the lower platform and its three legs is $m_{2}$, the moment equilibrium equation is established as

$$
x_{G 1} m_{1}-x_{G 3}\left(m_{1}+m_{2}\right)=0
$$

To sum up, the horizontal coordinate of the point $G_{3}$ is

$$
x_{G 3}=\frac{x_{G 1} m_{1}}{m_{1}+m_{2}}
$$

When the displacement of the upper platform is equal to zero, the projections of the two points $G_{1}$ and $G_{2}$ are coincident, too. If $G_{1}$ is located outside the supporting triangle, as shown in Figure 5b, the robot will lose its stability. The maximum step length of the robot is marked as $x_{G}$. Then, the step length of the robot, marked as $S$, can be expressed as:

$$
S \leq x_{G}
$$

Here, the maximum step length of the hexapod walking robot is calculated and analyzed in theory only according to the instability condition. The movement of the platform is restricted by the equivalent link length $l_{i}(i=1,2,3)$ of the limb, which may not reach the maximum step length before the instability.

\section{Gait Planning of 3-UPU Hexapod Walking Robot}

For the new walking robot, we develop a special type of gait, which is simple and easy to implement.

\subsection{A New Gait}

The walking of 3-UPU hexapod robot is realized by alternating motion between two platforms. A gait called 3-3 gait, i.e., alternate tripod support gait, is used in the process of walking. The legs are divided into two groups, in turn in the support phase or suspending phase state.

As the robot uses two groups of legs to walk, the duty factor $\beta$ is defined as 0.5 for the hexapod walking robot. This ensures that the robot has three legs on the ground to keep the body stable, while the robot has relatively high walking efficiency and moderate velocity. The gait diagram is displayed in Figure 6, where the thick line stands for the state when a leg is supporting on the ground and a thin line for legs in the air.

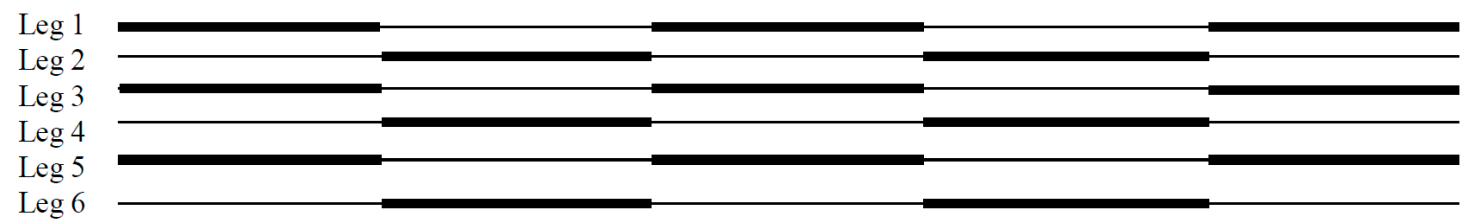

Figure 6. 3-3 gait diagram of 3-UPU hexapod walking robot.

The gait of one cycle in walking is divided into two stages:

Stage I: The six legs of the robot land on the ground in the initial state. To begin with, a group of legs-namely, legs 1,3,5-in the lower platform serves as a support, the upper platform lifts, as shown in Figure 7a; Then, the upper platform moves forward, as shown in Figure 7b; Finally, a group of legs, namely-legs 2, 4,6-in the upper platform lands on the ground as shown in Figure 7c.

Stage II: When a group of legs in the upper platform lands on the ground, the legs in the lower platform-namely, legs 1, 3 and 5-will be lifted, as shown in Figure 7d; Then the lower platform 
moves forward, as shown in Figure 7e; Finally, these legs land on the ground, as shown in Figure 7f. A cycle of this gait is completed.

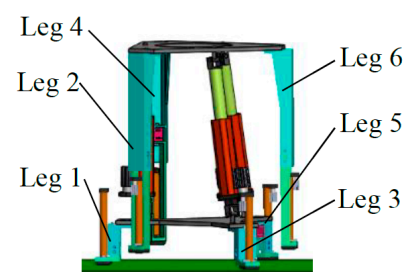

(a)

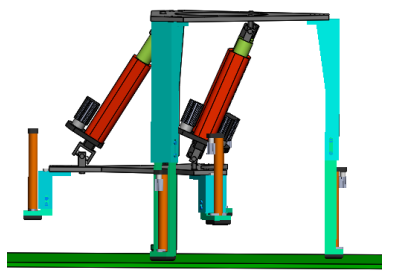

(d)

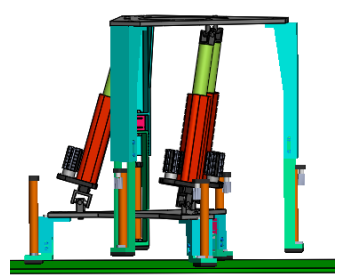

(b)

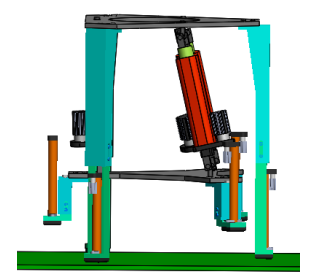

(e)

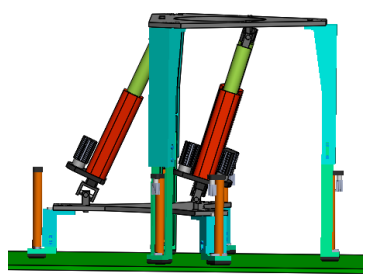

(c)

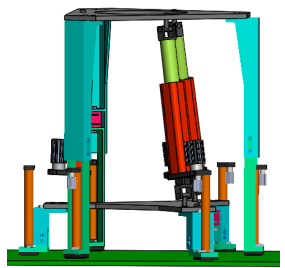

(f)

Figure 7. Walking gait of 3-UPU hexapod walking robot. (a) Lifting of the upper platform. (b) Moving forward of the upper platform. (c) Landing on the ground of the upper platform. (d) Lifting of the lower platform. (e) Moving forward of the lower platform. (f) Landing on the ground of the lower platform.

\subsection{Gait Parameters of 3-UPU Hexapod Walking Robot}

When the upper platform moves, according to the position inverse solution Equation (6), the parameter variation of each driving motor, i.e., the equivalent link length $l_{i}(i=1,2,3)$ of the limb $i$ can be expressed as:

$$
\left\{\begin{array}{l}
l_{1}=\sqrt{(x+\Delta)^{2}+y^{2}+z^{2}} \\
l_{2}=\sqrt{\left(x-\frac{1}{2} \Delta\right)^{2}+\left(y-\frac{\sqrt{3}}{2} \Delta\right)^{2}+z^{2}} \\
l_{3}=\sqrt{\left(x-\frac{1}{2} \Delta\right)^{2}+\left(y+\frac{\sqrt{3}}{2} \Delta\right)^{2}+z^{2}}
\end{array}\right.
$$

where $(x, y, z)$ is coordinate of the moving platform reference point $O^{\prime}$ in the static coordinate system $O-x y z$.

When the lower platform moves, according to the inverse solution equation of the position of the mechanism, the equivalent link length $l_{i}(i=1,2,3)$ of the limb $i$ can be obtained as:

$$
\left\{\begin{array}{l}
l_{1}=\sqrt{(x-\Delta)^{2}+y^{2}+z^{2}} \\
l_{2}=\sqrt{\left(x+\frac{1}{2} \Delta\right)^{2}+\left(y+\frac{\sqrt{3}}{2} \Delta\right)^{2}+z^{2}} \\
l_{3}=\sqrt{\left(x+\frac{1}{2} \Delta\right)^{2}+\left(y-\frac{\sqrt{3}}{2} \Delta\right)^{2}+z^{2}}
\end{array}\right.
$$

\subsection{Stride Calculation of 3-UPU Hexapod Walking Robot}

The hexapod walking robot is realized by alternating motion between the two platforms. Therefore, to avoid collision between the two groups of supporting legs, the maximum step size of the robot should be limited. In Figure $8 \mathrm{a}, \triangle L M N$ and $\triangle E F H$ represent the robot's upper and lower platforms, respectively. The small squares at six vertices represent six legs. Taking the upper platform starting to move as an example, point $L$ as the reference point, the equivalent radius of leg cross section is marked as $R_{T}$. When the upper platform moves forward along forward direction of $x$ axis, as shown 
in Figure 8b, the interference between the leg of $L, M$ and the leg of $F, H$, should be considered. On the premise of no collision, the expression of the maximum moving distance of the moving platform is:

$$
l_{\max 1}=L F-2 R_{T}=2\left(O J-R_{T}\right)=2\left(x_{G 3}-R_{T}\right)
$$

When the upper platform moves along $L H$ direction, as shown in Figure $8 \mathrm{c}$, the expression of the maximum moving distance of the moving platform is:

$$
l_{\max 2}=L P-R_{T}=x_{G 3}-R_{T}
$$

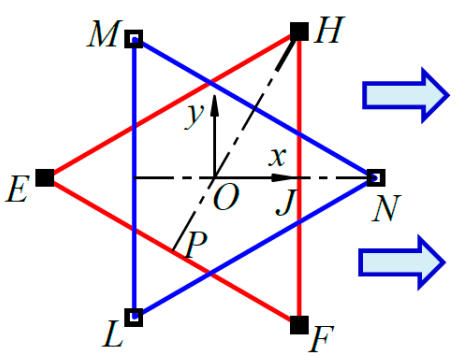

(a)

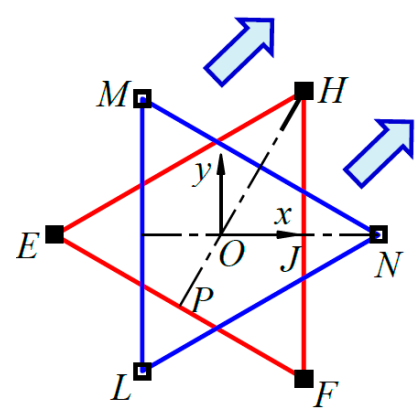

(c)

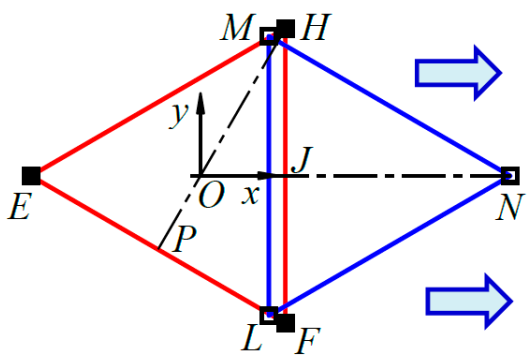

(b)

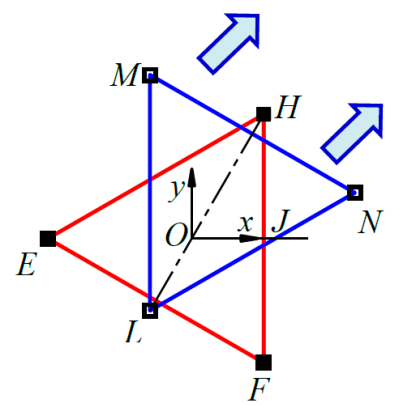

(d)

Figure 8. Motion diagram of 3-UPU hexapod walking robot. (a) Initial status. (b) Maximum moving distance of the upper platform along $x$ axis. (c) Initial status. (d) Maximum moving distance of the upper platform along $\mathrm{LH}$ direction.

The allowable maximum stride of the moving platform along forward direction of $x$ axis has to be larger than that when it moves along negative direction of $x$ axis, as demonstrated in Figure 8 . Combined with the analysis of the stability of the robot, the maximum stride of the robot is less than $x_{G 3}$ in the situation of without instability. In order to ensure the stability of the robot and avoid the interference of the legs, the maximum stride of the hexapod walking robot is set to $l_{\max 1}$. This situation occurs when the moving platform moves along the direction pointed by the triangle sharp angle of the supporting surface.

\subsection{Trajectories of the Two Platforms}

The trajectory of the moving platform is analyzed in related to walking on different terrains. When planning the gait of the hexapod walking robot, the trajectory curve of the moving platform can be divided into two kinds: straight line and curve. The trajectory of straight line is flexible at the same height of obstacle crossing, but at the turning point of the trajectory, the velocity and acceleration will change, which will affect the stability of the robot.

At the same obstacle height, the trajectory of the straight section is more flexible, but the mutation of the velocity and acceleration at the turning point of the trajectory occur, which affects the stability 
of the robot. A quadratic polynomial is thus adopted for the platform trajectory to avoid a sudden change of velocity and acceleration, whose expression is:

$$
z=a x^{2}+b x+c
$$

\section{Case Study}

We include a case study of the analysis of the 3-UPU parallel mechanism-based walking robot. The design parameters of the 3-UPU parallel mechanism are shown in Table 2.

Table 2. Design parameters of the 3-UPU parallel mechanism.

\begin{tabular}{cc}
\hline Parameter & Value \\
\hline Equivalent circumradius of the upper platform, $r / \mathrm{mm}$ & 125 \\
Equivalent circumradius of the lower platform, $R / \mathrm{mm}$ & 160 \\
Range of the equivalent link length of limb $i, l_{i}(i=1,2,3) / \mathrm{mm}$ & $l_{i} \in[365,465]$ \\
Range of the rotation angle of the Hooke joint, $\theta_{i}(i=1,2) / \mathrm{rad}$ & $\theta_{i} \in[-\pi / 4, \pi / 4]$ \\
\hline
\end{tabular}

Following the workspace search flow chart shown in Figure 3, the workspace of the 3-UPU parallel mechanism formed by the combination of the top and bottom surfaces and the surrounding surfaces, as shown in Figure 9. The upper and lower surfaces are formed by the limit position of the equivalent link length $l_{i}(i=1,2,3)$ of limb $i$. The surrounding surfaces are constrained by the limit position of the rotation angle $\theta_{i}$ of the Hooke joint. It was also found that the influence of the limit positions of two revolute pairs on the workspace is different.

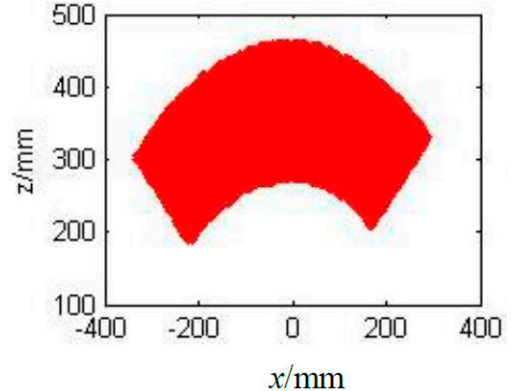

(a)

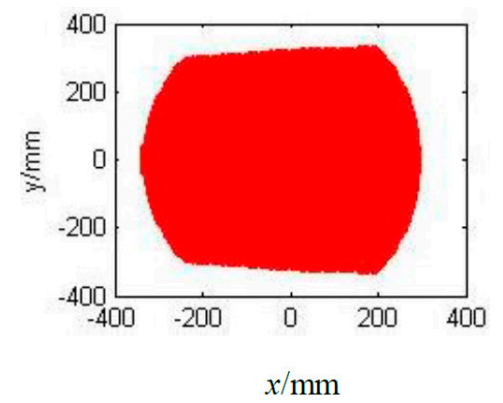

(c)

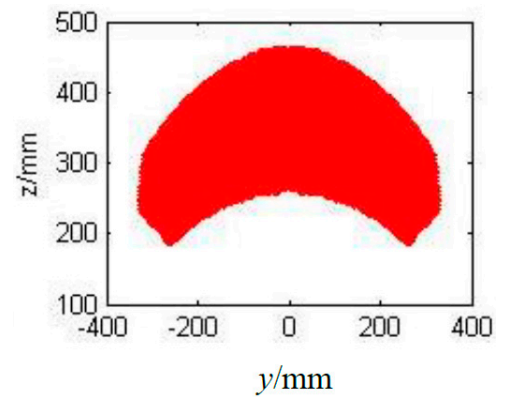

(b)

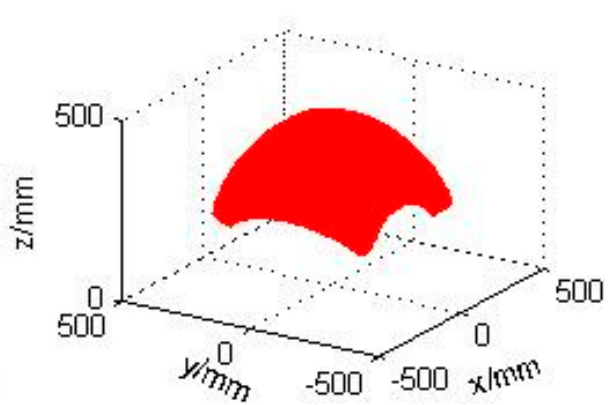

(d)

Figure 9. Workspace of the 3-UPU parallel mechanism. (a) Workspace projection in $x z$ plane. (b) Workspace projection in $y z$ plane. (c) Workspace projection in $x y$ plane. (d) 3D Workspace.

In the initial assembly, the coordinates of the upper platform center in the static coordinate system is $(0,0,363)$. The variation curves of the parameters of the limb lengths can be obtained when the upper platform moves using MATLAB simulation, as shown in Figure 10a,b. 


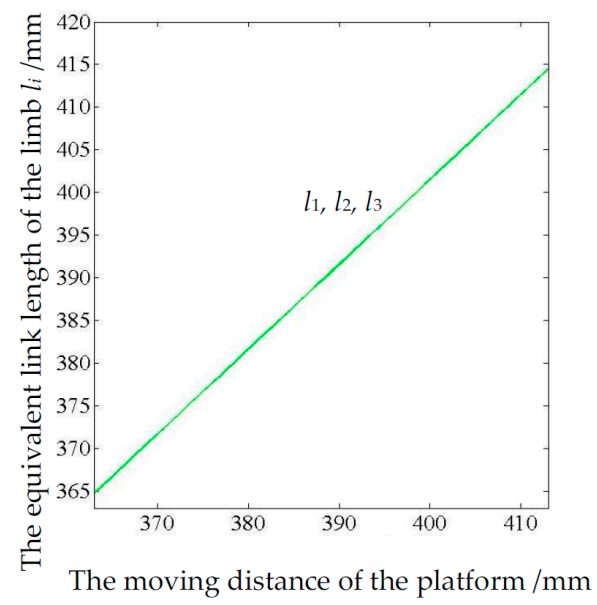

(a)

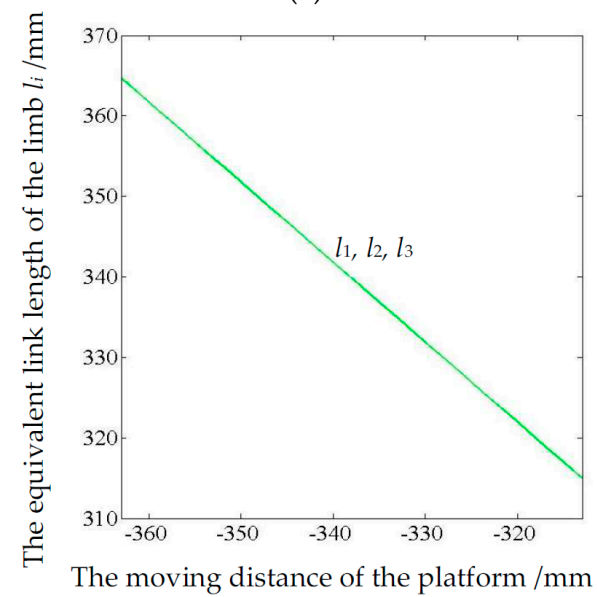

(c)

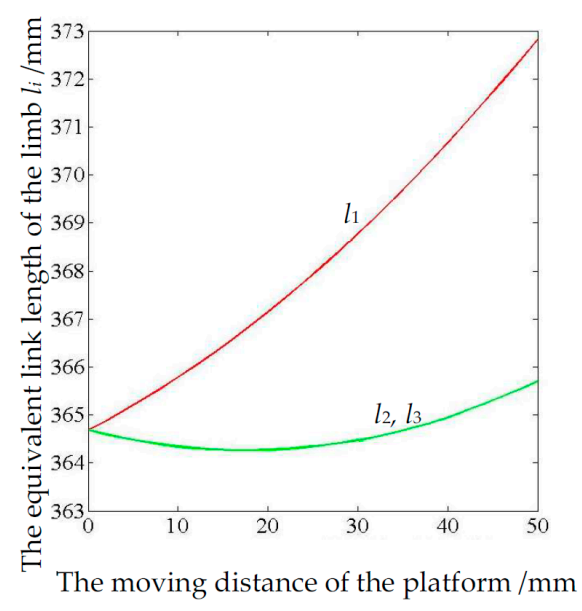

(b)

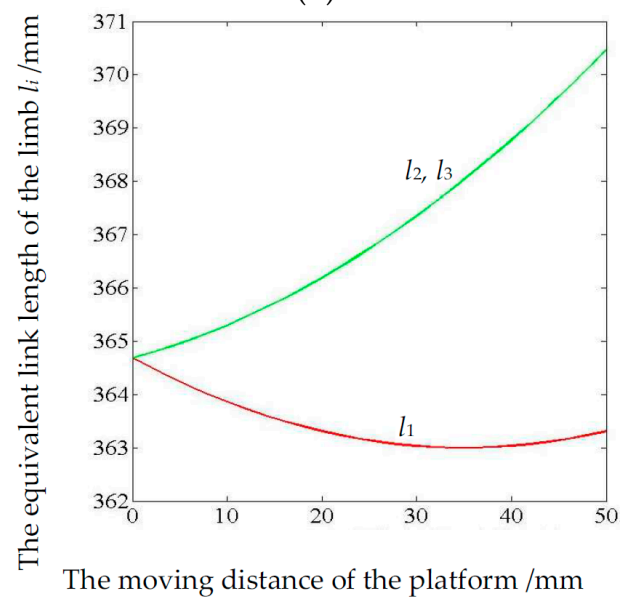

(d)

Figure 10. Variation of the limb lengths when the upper or lower platform moves. (a) The upper platform moves forward along $z$ axis. (b) The upper platform moves along $x$ axis. (c) The lower platform moves along $z$ axis. (d) The lower platform moves along $x$ axis.

When the lower platform moves, the moving and static coordinate systems exchange. The coordinate of the lower platform center in the static coordinate system is $(0,0,-363) \mathrm{mm}$. The variation curve of parameters of each driving motor can be obtained from Equation (6), as shown in Figure 10c,d. From Figure 10, the equivalent link length of each $\operatorname{limb} l_{i}(i=1,2,3)$ varies smoothly when the upper platform moves, that is, the displacement of each driving motor is stable.

When the upper platform is conducted as the moving platform, the static coordinate system is established in the center of the lower platform. According to the robot's gait planning, the maximum stride $l_{\max 1}$ is $160 \mathrm{~mm}$, the starting point coordinate of the curve is $(0,363)$, and the ending point coordinate is $(160,363)$. According to the workspace of the 3-UPU parallel mechanism, the maximum obstacle height of the robot could cross is $80 \mathrm{~mm}$, that is, the extreme point coordinate of the curve is $(80,443)$. Substituting the coordinates of the starting point, the end point and the extreme point into Equation (19), the trajectory equation of the reference point of the upper platform can be obtained,

$$
z=-0.0125 x^{2}+2 x+363
$$

Taking the lower platform as the moving platform, the static coordinate system is connected to the center of the upper platform. The maximum stride is $160 \mathrm{~mm}$. Substituting the starting point coordinate $(-60,-363)$, the ending point coordinate $(0,-363)$, and the extreme point coordinate $(-80$, 
-283) of the curve into Equation (19), the trajectory curve equation of the centroid of the reference point of the lower platform can be obtained as:

$$
z=-0.0125 x^{2}-2 x-363
$$

Following trajectory specified by Equation (21), walking along a straight line on flat terrain with the robot was simulated in SolidWorks. A snapshot is shown in Figure 11. The variation of the stability margin is shown in Figure 12. The stability margin is calculated by $d=\min \left\{d_{1}, d_{2}, d_{3}\right\}$, where $d_{i}, i=1,2$, 3 are the distances from $G_{3}$ to three sides of the supporting triangle, as demonstrated in Figure 5a. As can be seen in Figure 12, the stability margin $d$ is always larger than zero, thus static walking stability is guaranteed.

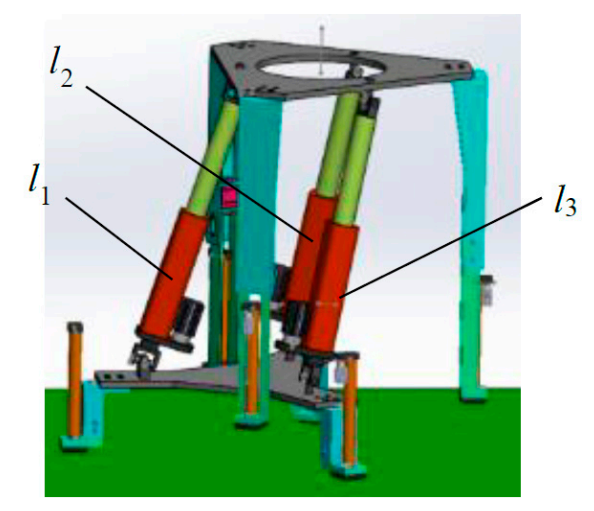

Figure 11. Walking on flat terrain of 3-UPU hexapod walking robot.

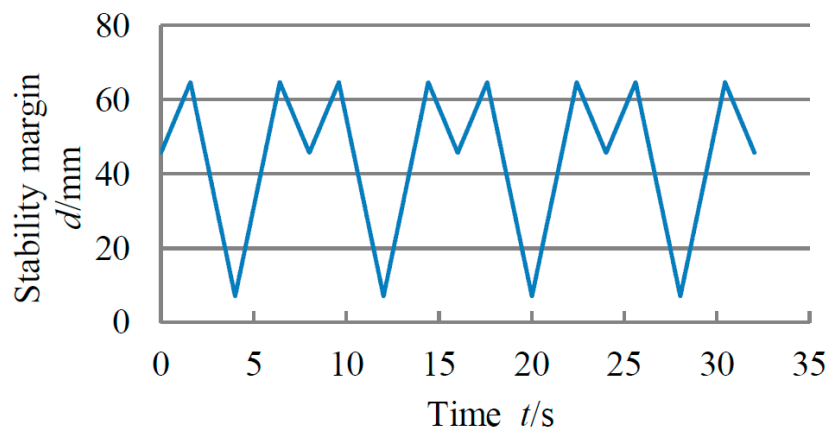

Figure 12. Variation of the stability margin when walking on flat terrain.

A simulation of walking on uneven terrain is shown in Figure 13, in which the robot walks over a ditch. The step length is adjusted to fit both the ditch width and also the stability requirement. The simulation shows that the robot can walk over the ditch stably, with the stability margin displaying in Figure 14. Compared with Figure 12 for even terrain walking, the stability margin (SM) plot in Figure 14 shows some differences. One is that the change of SM is not periodic, as the walking robot has to adjust its stride in ditch crossing. Another difference is that, in Figure 14, the SM for some short periods remains unchanged. This is because the two platforms do not move but only legs are extended to fit the terrain. It is noted that the SM in Figure 14 is always larger than zero too, which guarantees a stable static walking. 


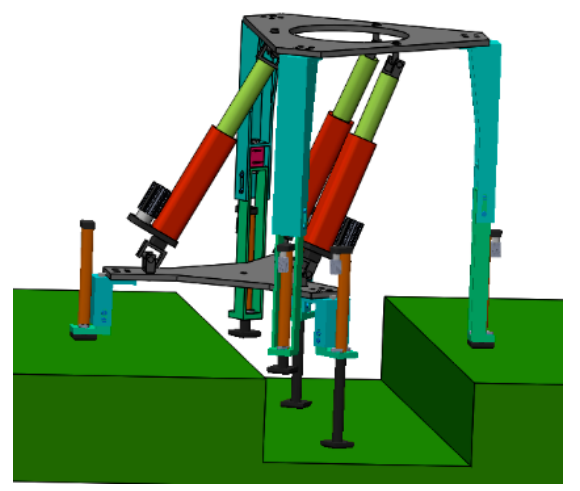

Figure 13. Crossing a ditch of $140 \mathrm{~mm}$ in depth and $350 \mathrm{~mm}$ in width by the 3-UPU hexapod walking robot.

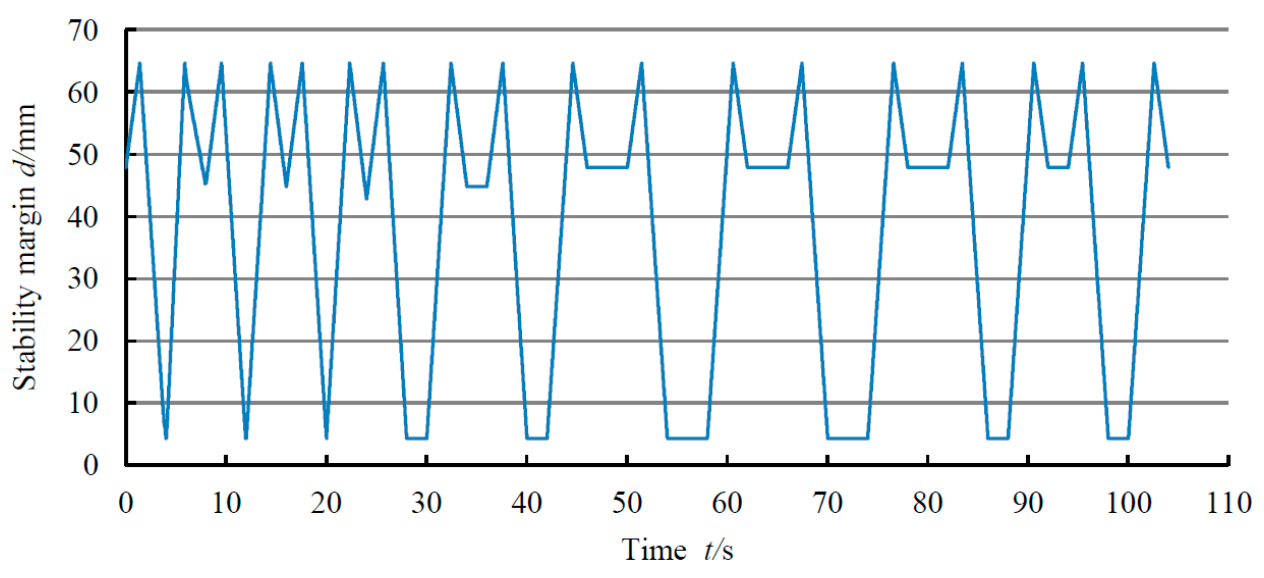

Figure 14. Variation of the stability margin when crossing ditch.

\section{Discussion and Conclusions}

A hexapod walking robot built with a 3-UPU parallel mechanism is introduced in this paper. The inverse kinematics solution of the mechanism platform is analyzed, the closed vector loop equation is established, and the inverse solution is obtained. The workspace of the mechanism is obtained using the constraint condition of each joint and the inverse kinematics solution.

The movement stability of the robot is analyzed using the center of gravity projection method. On the premise of maintaining the stability of the body, according to the structure parameters of the robot and without collision between the legs or between the legs and the body, the maximum transverse moving stride of the robot is $160 \mathrm{~mm}$. The gait planning of the hexapod walking robot is carried out, and the variation of the equivalent link length of each limb with the movement of the robot is obtained.

The novelty of the work lies in the introducing of the 3-UPU parallel mechanism in the walking robot. While parallel mechanisms were mostly used as legs in most works [17-21], in this work, we innovatively use the parallel mechanism as both an actuator to generate walking and also a connecting body to connect two groups of three legs. The new walking robot is thus able to walk with simple gait using very few motors. Moreover, the robot has a high terrain adaptability and stability, which has been demonstrated through simulations. The robot also has other potentials, including large carrying capacity and turning ability. In future work, we will focus on the detection of the robot's center of gravity position and study further on stable walking on other uneven terrains, such as slopes and stairs. Experimental investigation with a prototype under developed is also planned. 
Author Contributions: R.L. provided the ideas and wrote the paper manuscript. H.M. completed the stability and gait planning. S.B. contributed in walking analysis and major revision. Y.Y. and J.Z. tested the simulation. All authors read and approved the final manuscript.

Funding: This research was funded by the National Natural Science Foundation of China (grant number 51275486) and the Foundation of Shanxi Key Laboratory of Advanced Manufacturing Technology (grant number XJZZ201702).

Conflicts of Interest: The authors declare no conflict of interest.

\section{References}

1. Tedeschi, F.; Carbone, G. Design issues for hexapod walking robots. Robotics 2014, 3, 181-206. [CrossRef]

2. Yang, F.; Ding, X.L.; Peng, S.J. Bio-control of a modular design robot-NOROS. In Proceedings of the Third ASME/IFToMM International Conference on Reconfigurable Mechanisms and Robots, Beijing, China, 20-22 July 2015; pp. 891-900.

3. Peng, S.J.; Ding, X.L.; Yang, F.; Xu, K. Motion planning and implementation for the self-recovery of an overturned multi-legged robot. Robotica 2017, 35, 1107-1120. [CrossRef]

4. Ma, Z.R.; Guo, W.Z.; Gao, F. Analysis on obstacle negotiation ability of a new wheel-legged robot. Mach. Des. Res. 2015, 31, 6-10, 15. (In Chinese)

5. Tsai, L.W. Kinematics of a three-DOF platform with three extensible limbs. In Recent Advances in Robot Kinematics; Lenarcic, J., Parenti-Castelli, V., Eds.; Kluwer Academic: London, UK, 1996; pp. 401-410.

6. Gregorio, R.D. Kinematics of the 3-UPU wrist. Mech. Mach. Theory 2003, 38, 253-263. [CrossRef]

7. Ji, P.; Wu, H.T. Kinematics analysis of an offset 3-UPU translational parallel robotic manipulator. Robot. Auton. Syst. 2003, 42, 117-123. [CrossRef]

8. Han, C.; Kim, J.; Kim, J.; Park, F.C. Kinematic sensitivity analysis of the 3-UPU parallel mechanism. Mech. Mach. Theory 2002, 37, 787-798. [CrossRef]

9. Huang, Z.; Li, S.H.; Zuo, R.G. Feasible instantaneous motions and kinematic characteristics of a special 3-DOF 3-UPU parallel manipulator. Mech. Mach. Theory 2004, 39, 957-970. [CrossRef]

10. Wu, T.; Zhang, W.X.; Ding, X.L. Design and analysis of a novel parallel metamorphic mechanism. J. Mech. Eng. 2015, 51, 30-37. (In Chinese) [CrossRef]

11. Staicu, S.; Popa, C. Kinematics of the spatial 3-UPU parallel robot. UPB Sci. Bull. Ser. D 2013, 75, 9-18.

12. Lu, Y.; Shi, Y.; Hu, B. Kinematic analysis of two novel 3UPU I and 3UPU II PKMs. Robot. Auton. Syst. 2008, 56, 296-305. [CrossRef]

13. Staicu, S.; Popa, C. Dynamics of the translational 3-UPU parallel manipulator. UPB Sci. Bull. Ser. D 2014, 76, 3-12.

14. Gregorio, R.D.; Parenti-Castelli, V. Mobility analysis of the 3-UPU parallel mechanism assembled for a pure translational motion. In Proceedings of the IEEE/ASME International Conference on Advanced Intelligent Mechatronics (AIM), Atlanta, GA, USA, 19-23 September 1999; pp. 520-525.

15. Peng, B.B.; Li, Z.M.; Wu, K.; Sun, Y. Kinematic characteristics of 3-UPU parallel manipulator in singularity and its application. Int. J. Adv. Robot. Syst. 2011, 8, 54-64.

16. Zhang, D.; Wei, B. Interactions and optimizations analysis between stiffness and workspace of 3-UPU robotic mechanism. Meas. Sci. Rev. 2017, 17, 83-92. [CrossRef]

17. Miao, Z.H.; Yao, Y.A.; Kong, X.W. A rolling 3-UPU parallel mechanism. Front. Mech. Eng. 2013, 8, 340-349. [CrossRef]

18. Gu, Q.F. Kinematics of 3-UPU parallel leg mechanism used for a quadruped walking robot. In Proceedings of the 14th International Symposium on Distributed Computing and Applications for Business Engineering and Science (DCABES 2015), Guiyang, China, 18-24 August 2015; pp. 188-191.

19. Wang, M.F.; Ceccarelli, M.; Carbone, G. A feasibility study on the design and walking operation of a biped locomotor via dynamic simulation. Front. Mech. Eng. 2016, 11, 144-158. [CrossRef]

20. Sugahara, Y.; Endo, T.; Lim, H.; Takanishi, A. Design of a battery-powered multi-purpose bipedal locomotor with parallel mechanism. In Proceedings of the IEEE/RSJ International Conference on Intelligent Robots and Systems, Lausanne, Switzerland, 30 September-4 October 2002; pp. 2658-2663.

21. Wang, H.B.; Qi, Z.Y.; Hu, Z.W.; Huang, Z. Application of parallel leg mechanisms in quadruped/biped reconfigurable walking robot. J. Mech. Eng. 2009, 45, 24-30. (In Chinese) [CrossRef] 
22. Hirakoso, N.; Terayama, J.; Yoshinaga, N.; Arai, T. A study on optimal formulation for multi-legged gait robot with redundant joint. In Proceedings of the International Symposium on Micro-Nanomechatronics and Human Science, Nagoya, Japan, 3-6 December 2017; pp. 1-6.

23. Sun, T.; Xiang, X.; Su, W.H.; Wu, H.; Song, Y.M. A transformable wheel-legged mobile robot: Design, analysis and experiment. Robot. Auton. Syst. 2017, 98, 30-41. [CrossRef]

24. Bai, S.; Low, K.H.; Zielinska, T. Quadruped free gait generation for straight-line and circular trajectories. Adv. Robot. 2000, 13, 513-538. [CrossRef]

25. Bai, S.; Low, K.H. Terrain evaluation and its application to path planning for walking machines. Adv. Robot. 2001, 15, 729-748. [CrossRef]

26. Bai, S.; Low, K.H.; Teo, M.Y. Path generation of walking machines in $3 \mathrm{D}$ terrain. In Proceedings of the IEEE International Conference on Robotics and Automation, Washington, DC, USA, 11-15 May 2002; pp. 2216-2221.

27. Gong, D.W.; Wang, P.; Zhao, S.Y.; Duan, Y. Bionic quadruped robot dynamic gait control strategy based on twenty degrees of freedom. IEEE/CAA J. Autom. Sin. 2018, 5, 382-388. [CrossRef]

28. Li, M.T.; Jiang, Z.Y.; Wang, P.F.; Sun, L.N.; Ge, S.S. Control of a quadruped robot with bionic springy legs in trotting gait. J. Bionic Eng. 2014, 11, 188-198. [CrossRef]

29. Matsuzawa, T.; Koizumi, A.; Hashimoto, K.; Sun, X.; Hamamoto, S.; Teramachi, T.; Sakai, N.; Kimura, S.; Takanishi, A. Crawling motion and foot trajectory modification control for legged robot on rough terrain. In Proceedings of the IEEE International Conference on Mechatronics and Automation, Takamatsu, Japan, 6-9 August 2017; pp. 1976-1982.

30. Winkler, A.W.; Farshidian, F.; Pardo, D.; Neunert, M.; Buchli, J. Fast trajectory optimization for legged robots using vertex-based ZMP constraints. IEEE Robot. Autom. Lett. 2017, 2, 2201-2208. [CrossRef]

31. Winkler, A.W.; Bellicoso, C.D.; Hutter, M.; Buchli, J. Gait and trajectory optimization for legged systems through phase-based end-effector parameterization. IEEE Robot. Autom. Lett. 2018, 3, 1560-1567. [CrossRef]

32. Neunert, M.; Farshidian, F.; Winkler, A.W.; Buchli, J. Trajectory optimization through contacts and automatic gait discovery for quadrupeds. IEEE Robot. Autom. Lett. 2017, 2, 1502-1509. [CrossRef]

33. Zhao, Y.; Chai, X.; Gao, F. Obstacle avoidance and motion planning scheme for a hexapod robot Octopus-III. Robot. Auton. Syst. 2018, 103, 199-212. [CrossRef]

34. Oliveira, L.F.P.; Rossini, F.L. Modeling, simulation and analysis of locomotion patterns for hexapod robots. IEEE Lat. Am. Trans. 2018, 16, 375-383. [CrossRef] 